Cinémas

Revue d'études cinématographiques

Journal of Film Studies

Sean CUBITT, The Cinema Effect, Cambridge/London, MIT

Press, 2004, 456 p.

\title{
Jan Baetens
}

Volume 15, numéro 1, automne 2004

Entre l’Europe et les Amériques

URI : https://id.erudit.org/iderudit/011664ar

DOI : https://doi.org/10.7202/011664ar

Aller au sommaire du numéro

Éditeur(s)

Cinémas

ISSN

1181-6945 (imprimé)

1705-6500 (numérique)

Découvrir la revue

Citer ce compte rendu

Baetens, J. (2004). Compte rendu de [Sean CUBITT, The Cinema Effect, Cambridge/London, MIT Press, 2004, 456 p.] Cinémas, 15(1), 163-167. https://doi.org/10.7202/011664ar d'utilisation que vous pouvez consulter en ligne.

https://apropos.erudit.org/fr/usagers/politique-dutilisation/ 
Sean CUBITT, The Cinema Effect, Cambridge/London, MIT Press, 2004, 456 p.

The Cinema Effect est sans doute l'un des livres les plus ambitieux sur le cinéma qui aient paru ces dernières années. À la manière du grand diptyque de Gilles Deleuze, L'Imagemouvement (1983) et L'Image-temps (1985), l'ouvrage de Cubitt se présente en effet comme une véritable théorie d'ensemble du fait cinématographique, qui tente d'allier une interprétation philosophique englobante - très marquée par la sémiotique de Peirce - à un parcours historique aussi complet que possible. Les analogies avec le travail de Deleuze ne doivent cependant pas masquer de réelles divergences, qu'expliquent, d'une part, les mutations du cinéma et, d'autre part, les transformations au sein des recherches universitaires. Le cinéma du XXI ${ }^{\mathrm{e}}$ siècle n'est plus «national» ni "occidental» : il est devenu «mondial», et les objets sur lesquels se penche Cubitt, tout comme les enjeux que son approche soulève, s'en ressentent. Dans son ouvrage, une large place est donc occupée par le cinéma non occidental et la problématique du cinéma "cosmopolite». De même, le discours sur le cinéma s'y éloigne, comme cela est généralement le cas actuellement, des grandes taxinomies formelles du structuralisme, pour s'ouvrir davantage au contexte social, voire, dans l'analyse néomarxiste de l'auteur, au cinéma comme marchandise.

Dans The Cinema Effect, ces divers horizons et multiples influences se retrouvent dans une synthèse étourdissante, au double sens du terme. Car Cubitt fascine autant qu'il laisse perplexe et son livre ne suscite pas moins l'admiration que l'agacement. Les trois sections du livre («Pioneer Cinema», soit le cinéma des premier temps; "Normative Cinema», soit le cinéma de l'âge classique, celui de l'image-mouvement 
deleuzien ; "Post-Cinema ", terme américain très populaire dont le sens est ici nettement plus vaste que celui de "cinéma numérique " auquel certains ont tendance à le réduire: "cinéma postmoderne» en paraît un équivalent plus acceptable), puis les quatorze chapitres presque tous centrés sur l'analyse d'un film ou d'un auteur particuliers, enfin les quelques dizaines de lectures, parfois rapides, parfois plus détaillées, disséminées dans tout l'ouvrage, n'emportent pas tous l'adhésion de la même manière. Par moments, le lecteur renâcle même fortement. Toutefois, l'impression globale que laisse le livre est positive. D'abord, certes, en raison de son culot théorique, ensuite à cause de la finesse et de l'ingéniosité de nombreuses analyses microscopiques, enfin grâce à l'indignation morale et politique qui traverse le livre d'un bout à l'autre. Bref, pour contestables que paraissent bien de ses idées, Cubitt offre ici un livre puissant, stimulant, provocant.

S'appuyant sur la triade peircienne de «firstness" (la chose en soi), "secondness" (la chose en tant qu'elle se rapporte à quelque chose d'autre) et "thirdness" (la chose en tant qu'elle représente quelque chose à la manière d'un signe), Cubitt distingue trois grands modes d'existence de l'image, qui définissent chacun des effets spécifiques qu'il caractérise à l'aide de métaphores empruntées à la culture digitale: le "pixel» (l'image mobile telle qu'en elle-même), le "cut" (le montage, qui insère le pur déroulement du temps dans un dispositif spatial) et le "vector» (l'image comme pure création symbolique, détachée du réel et seulement déchiffrable par un acte interprétatif). Pour simple et séduisante qu'elle soit à première vue, ne fût-ce que parce qu'elle rompt avec les habituelles dichotomies de la théorie du cinéma ("cinéma des premiers temps» versus «cinéma narratif" ou "image-mouvement" versus "image-temps ", pour ne citer que les oppositions les plus répandues), la triade en question devient très vite problématique par l'usage ou, plus exactement, par le non-usage qu'en fait l'auteur. En effet, le cadre épistémologique de Peirce n'est présent qu'en toile de fond, et souvent de manière strictement implicite: une fois sa grande triade posée, Cubitt n'y revient plus que très rarement, de sorte que la plus-value méthodologique et théorique du 
système peircien n'est ni mise en lumière ni mise à l'épreuve. Le mode dominant des lectures concrètes de l'auteur n'est en tout cas nullement peircien, la grande architecture du livre étant donnée par le métarécit de la culture comme marchandise, qui suit pas à pas l'évolution du système économique capitaliste. De plus, les trois pôles du "pixel», du «cut» et du «vector» font l'objet d'une interprétation pour le moins essentialisante. Chaque fois, une seule œuvre suffit à illustrer la logique d'un des trois modes de l'image: les premiers documentaires des frères Lumière le font pour le "pixel", les films à trucages de Méliès pour le "cut» et Fantasmagorie, un dessin animé moins connu d'Emile Cohl, pour le "vector». Les possibilités de mélanges et d'imbrications des trois pôles sont quant à elles soigneusement écartées, sauf à la toute fin du livre, lorsque Cubitt, pris d'un repentir un peu tardif, reconnaît la possibilité d'un traitement différent de "pixel ", "cut" et "vector" (p. 360). Pareille analyse, qui excède le cloisonnement des trois types, est pourtant la seule qui soit compatible avec la pensée de Peirce: refuser les caractéristiques du "second" ou du "troisième" à L'Arrivée d'un train en gare de la Ciotat ou à La Sortie des usines Lumière est un parti pris théorique difficilement défendable. Il faut encore ajouter à cela, et c'est là que les choses se gâtent vraiment, que la triade initiale tend à proliférer et à s'agglutiner, dans une sorte de libre association, avec d'autres systèmes ternaires: Réel-Imaginaire-Symbolique, RéférentSignifiant-Signifié, Préindividuel-Individuel-Social, Absence de temps-Destin-Espoir, et j'en passe (pour un aperçu, voir p. 9798). Cette manie totalisatrice, dont l'auteur tient pourtant à se démarquer coûte que coûte (il va jusqu'à faire appel au couple "totalité/infinité » de Lévinas dans ce but!), le conduit à plus d'une assertion imprudente. Je pense par exemple à l'interprétation éminemment idéologique de la notion de récit comme instance de hiérarchisation antidémocratique de la "priméité" visuelle des films des Lumière (voir p. 35-41). Le goût des schémas universels le pousse également à commettre des lectures monolithiques dont on peut croire qu'elles font violence à leurs objets. L'idée de purger les films des Lumière de toute ce qu'on pourrait y trouver d'étranger à la dimension du "premier» en 
fournit un exemple parlant. Enfin, le même désir de totalisation entrave régulièrement le fil de l'argumentation même de Cubitt, vite enclin à écraser son lecteur de références savantes (on saute de Lacan à Heidegger, de Frege à Crary, de Levinas à Silverman, sans oublier l'inévitable principe d'incertitude...) et à se laisser aller à des formules apodictiques qu'on croyait abandonnées dans la prose académique depuis Marshall McLuhan. Certes, cela fait joli d'accumuler des phrases du genre: "Cinematic spatialization has opted for architecture, while the world has chosen geography. In the interstices lies the possibility of a new art, made from the raw materials of time» (p. 364), mais elles n'épateront, malgré le brio du style, que le lecteur demi-savant.

Ces imperfections sont d'autant plus regrettables que Cubitt a vraiment des choses à dire sur le cinéma, même si les grandes lignes de son livre n'apprennent rien de vraiment neuf au lecteur, comme on le voit bien dans les trois chapitres sur le cinéma "normatif", respectivement consacrés à Alexandre Nevsky, à La Règle du jeu et au studio RKO. Ces analyses se contentent de reformuler nos connaissances sur ce qu'on appelait naguère les cinémas russe, français et américain de l'entredeux-guerres. Cela dit, les lectures ne manquent jamais de finesse ni de perspicacité; elles arrivent aussi à bien mettre en perspective, en les replaçant dans leur contexte historique, la production comme la réception des films, et elles portent enfin un regard souvent subtil sur la structure d'ensemble et sur les modes de fonctionnement précis des œuvres passées en revue. (Cependant, ici encore, le démon de la triade continue à chatouiller l'auteur; voir à cet effet le tableau récapitulatif, p. 188.)

La partie la plus intéressante du livre est la troisième, où Cubitt analyse justement les tensions et les contradictions du cinéma post-classique, tout en établissant des correspondances très convaincantes avec l'arrière-fond socioéconomique de l'industrie du cinéma (le corpus analysé va ici de Sam Peckinpah à Crouching Tiger, Hidden Dragon, en passant par de nombreux blockbusters des années 1980 et 1990). Dans un très beau chapitre sur le film "néobaroque», Cubitt renouvelle de fond en comble la vision convenue du néobaroque comme synonyme de dispersion postmoderne (comme c'est notamment le cas dans le 
livre d'Omar Calabrese, Neo-Baroque: A Sign of the Times). Confrontant aussi bien des techniques visuelles (comme le recours acrobatique à la caméra mobile) que narratives (comme l'utilisation de la logique narrative "paradigmatique» des banques de données, dont Lev Manovich propose une théorie dans The Language of New Media) et ontologiques (comme la représentation hyperréaliste de mondes impossibles), Cubitt montre que dans des films comme Batman (Tim Burton), Strange Days (Kathryn Bigelow) ou Snake Eyes (Brian de Palma), la dispersion des repères visuels sert moins à dérouter le public qu'à l'enfermer dans l'enceinte d'une cohésion illusoire où le récit, le temps, l'histoire, la société et, finalement, le monde, sont évacués au profit d'un mouvement purement spatial. On retrouve ici, quand bien même il n'est pas explicitement cité, les imprécations de Jameson contre l'effacement du Temps dans la Spatialité postmoderne. Dans le chapitre sur le cinéma "cosmopolite», c'est-à-dire destiné à un public mondial (et non plus seulement occidental ou oriental), Cubitt aborde des questions passionnantes sur les stratégies de marketing de ce genre de cinéma. Il y résume aussi l'une des thèses centrales du livre: les effets du cinéma contemporain sur le spectateur postmoderne; ce spectateur devient en effet lui-même marchandise, c'est-à-dire une donnée analysée, traitée, vendue dans la nouvelle économie d'un réseau informatique global. Comme l'auteur a le bon sens de ne pas croire à la fin de l'Histoire, The Cinema Effect se termine cependant par quelques pages où s'esquisse une première stratégie de résistance.

Jan Baetens

Katholieke Universiteit Leuven 